\title{
PERFIL PSICOPATOLÓGICO DIFERENCIAL DE LAS MUJERES LUDÓPATAS: IMPLICACIONES PARA EL TRATAMIENTO
}

\author{
Paz de Corral \\ Enrlque Echeburúa \\ Mayalen Irureta
}

Facultad de Psicologia. UNIVERSIDAD DEL PAÍs VASCO

\section{RESUMEN}

En los últimos años ha habido un interés creciente por los aspectos psicopatologicos y terapéuticos de la ludopatia. Sin embargo, la mayor parte de las investigaciones han tenido como participantes a hombres ludópatas. Además, ha habido pocos estudlos sobre las diferenclas de género en la ludopatfa, a pesar de que un terclo del total de ludópatas son mujeres. Los hombres manifiestan una preferencia por las máquinas recreativas, tienen frecuentemente un historial de abuso de alcohol o de conductas antisociales y muestran rasgos de impulsividad o de búsqueda de sensaciones. Las mujeres, por el contranio, se sienten más atraídas por los bingos, y los estados emocionales negativos desempeñan un papel importante en el inicio de la conducta de juego. Las mujeres juegan a menudo para escapar de los problemas o hacer frente a la mala relación de pareja o a la soledad y pueden tener

Correspondencia: Facultad de Psicología. Universidad del Pals Vasco. Avda. de Tolosa, 70. 20018 San Sebastián. 
antecedentes de abuso sexual o maltrato fisico en la infancia. Las mujeres se inlcian más tarde en el juego, pero se vuelven adictas más rápidamente. Las mujeres ludópatas responden mejor al tratamiento s/ se toman en cuente estas caracter/stlcas diferenciales. Por ello, parece haber diferenclas de género en el perfil clínico de los ludopatas, que deben tomarse en consideración en el diseño del tratamiento.

Palabras clave: JUEgo PATOLÓGICO; DIFERENCIAS dE GÉNERO; PSICOPATOLOGIA; IMPLICACIONES PARA EL TRATAMIENTO.

\section{SUMMARY}

Over the last few years there has been an increasing interest in the phenomenology and treatment of pathological gambling. However, to date, most research has been conducted in samples composed of predominantly or exclusively male pathological gamblers. In addition, little empinical research has evaluated gender differences in this disorder, in spite of women being one third of pathological gamblers. Male are attracted to slot machines, have a more frequent history of alcohol abuse or antisocial traits and tend to be impulsive and sensation seekers. However, women have a preference for bingos and slot machines and negative emotional states appear to play a bigger role tan in men in the gambling behavior. Women often gamble to escape from problems, troubled marniages, and loneliness, and have a higher frequency of history of abuse in childhood. Women start to gamble at a later age then men, but develop the disorder more quickly. Female pathological gamblers may respond better to treatment strategies that take more into account their emotional needs. There appear to be some gender differences in the clinical features of pathologlcal gambling dlsorders, and these differences may have treatment Implications.

Key words: PATHOLOGICAL GAMBLING; GENDER DIFFERENCES; PSYCHOPATHOLOGY; THEATMENT MPLICATIONS. 


\section{INTRODUCCIÓN}

La presencia incontrolada de máquinas tragaperras en bares y lugares de ocio, junto con la oferta abundante de bingos, casinos y de los juegos más tradicionales (quinielas, loterías, cupones, carreras de caballos, etcétera), ha hecho aumentar considerablemente la ludopatía. La indefensión ante esta avalancha de juegos con apuestas afecta especialmente a los adolescentes y a las personas más vulnerables psicológicamente.

En España la tasa de prevalencia de la ludopatía oscila entre el $2 \%$ y el $3 \%$ de la población adulta (Becoña, 1999; Irurita, 1996; Tejeiro, 1996). El trastomo es mucho más frecuente en hombres que en mujeres, pero éstas son mucho más reacias a buscar ayuda terapéutica por la censura social existente. A diferencia de otras conductas adictivas, el juego patológico se distribuye por todas las clases sociales y por todas las edades. No obstante, la edad de acceso al juego ha descendido en los últimos años. De hecho, cada vez son más los adolescentes que buscan tratamiento por problemas de juego (Báez y Echeburúa, 1995; Becoña, 2001).

Los ludópatas, al menos en España, muestran una dependencia fundamentalmente a las máquinas tragaperras, ya sea sólo a éstas o en combinación con otros juegos. La dependencia en exclusiva a otros juegos de azar es mucho menor (Báez, Echeburúa y Femández-Montalvo, 1994). Este hecho no es fruto de la casualidad, sino que obedece a una serie de aspectos psicológicos implicados en el funcionamiento de este tipo de máquinas (Echeburúa, 1992): su amplia difusión; el importe bajo de las apuestas con posibilidad de ganancias proporcionalmente cuantiosas; la brevedad del plazo transcurrido entre la apuesta y el resultado; la manipulación personal de la máquina (que genera una cierta ilusión de control); y las luces intermitentes de colores, que, junto con la música y el tintineo estrepitoso de las monedas, suscitan una tensión emocional y una gran activación psicofisiológica.

Desde una perspectiva psicopatológica, la depresión y el abuso de alcohol están asociados con frecuencia al juego patológico (Báez et al., 1994; McCormick, Russo, Ramírez y Taber, 1984). Por ello, la tasa de prevalencia del consumo abusivo de alcohol u otras drogas 
Tabla 1.- Estudios sobre mujeres ludopatas

\begin{tabular}{|c|c|c|}
\hline AUTORES & AÑo & MUESTRA \\
\hline Trevorrow \& Moore & 1998 & $\mathrm{~N}=95$ mujeres \\
\hline Arbinaga & $\begin{array}{l}2000 \\
2001 \\
\end{array}$ & $\begin{array}{c}\mathrm{N}=616 \\
308 \text { varones } \\
308 \text { mujeres }\end{array}$ \\
\hline $\begin{array}{c}\text { Getty, Watson \& Ron } \\
\text { Frisch }\end{array}$ & 2000 & $\begin{array}{c}N=60 \\
40 \text { varones } \\
20 \text { mujeres } \\
\end{array}$ \\
\hline $\begin{array}{l}\text { Tavares, Zilberman, } \\
\text { Beites \& Gentil }\end{array}$ & 2001 & $\begin{array}{c}\mathrm{N}=78 \\
38 \text { varones } \\
39 \text { mujeres }\end{array}$ \\
\hline Hing \& Breen & 2001 & $\begin{array}{c}N=2430 \\
1422 \text { varones } \\
1008 \text { mujeres }\end{array}$ \\
\hline Ladd \& Petry & 2002 & $\begin{array}{c}N=115 \\
70 \text { varones } \\
45 \text { mujeres }\end{array}$ \\
\hline Grant \& Suck von Kim & 2002 & $\begin{array}{c}\mathrm{N}=131 \\
53 \text { varones } \\
77 \text { mujeres }\end{array}$ \\
\hline $\begin{array}{l}\text { Ibáñez, Blanco, } \\
\text { Moreryra \& Saiz }\end{array}$ & 2003 & $\begin{array}{c}\mathrm{N}=69 \\
47 \text { varones } \\
22 \text { mujeres }\end{array}$ \\
\hline $\begin{array}{c}\text { Tavares, Martins, Lobo, } \\
\text { Silveira, Gentil \& } \\
\text { Hodging }\end{array}$ & 2003 & $\begin{array}{c}\mathrm{N}=140 \\
70 \text { varones } \\
70 \text { mujeres }\end{array}$ \\
\hline $\begin{array}{l}\text { Martins, Tavares, Lobo, } \\
\text { Galetti \& Gentil }\end{array}$ & 2004 & $\begin{array}{c}\mathrm{N}=156 \\
78 \text { varones } \\
78 \text { mujeres }\end{array}$ \\
\hline Petry \& Steinberg & 2005 & $\begin{array}{c}\mathrm{N}=149 \\
72 \text { varones } \\
77 \text { mujeres }\end{array}$ \\
\hline
\end{tabular}


entre los ludópatas oscila alrededor del 15\%-20\% (Báez et al., 1994; Lesieur y Heineman, 1988; Rodríguez-Martos, 1989).

El estudio de la ludopatía en función del sexo se ha hecho de una forma muy incompleta. De hecho, son muy pocas las mujeres que han formado parte de las investigaciones clínicas publicadas hasta la fecha (tabla 1). Las diferencias de la mujer respecto al hombre en el ámbito del juego patológico se manifiestan en diversos planos: los tipos de juego implicados, el mayor control en la obtención de dinero para jugar, la adquisición y el desarrollo del trastorno, el mayor nivel de psicopatología asociada y las repercusiones psicológicas más acentuadas en la familia. En concreto, el rechazo de la pareja hacia la mujer jugadora es mucho más explícito que en el caso inverso, así como su faita de colaboración en el proceso terapéutico. Asimismo hay una doble moral social ante el juego de la mujer. Asl como al hombre se le tolera el juego excesivo en las primeras fases, a la mujer se la tilda rápidamente de viciosa, lo que conlleva una ocultación del problema más tenaz, al menos extemamente, $y$, por ello, una resistencia a la búsqueda de ayuda terapéutica.

En suma, los ludópatas de uno y otro sexo se caracterizan por una dependencia emocional del juego, por una pérdida de control y por una interferencia negativa en el funcionamiento normal de la vida cotidiana, pero, sin embargo, adoptan perfiles diferenciales en función del sexo.

\section{CARACTERISTICAS DEMOGRÁFICAS}

De los ludópatas detectados en los estudios epidemiológicos en la población general, las mujeres constituyen el $30 \%$ del total. Sin embargo, en los centros clínicos de tratamiento de la ludopatía las mujeres representan el $10 \%-15 \%$ del total de los pacientes tratados. Hay, por tanto, una bolsa oculta de mujeres ludópatas que no consultan por su trastorno o que lo hacen tardiamente, cuando la situación es ya grave y cuando el pronóstico resulta más sombrío. Sin embargo, las mujeres son acompañantes frecuentes de los ludópatas cuando éstos deciden buscar ayuda. Así, el $82 \%$ de los ludópatas que acuden 
a los centros clínicos tienen como acompañantes a mujeres (pareja o madre, fundamentalmente) (Arbinaga, 2001).

En cuanto a la edad, hay dos picos significativos: una edad joven (en torno a los 18-30 años) y una edad más tardía (en torno a los 45-55 años). El estado civil está directamente relacionado con la edad. Hay un predominio de solteras en el primer tramo de edad y de casadas en el segundo, si bien el número de divorciadas en este último tramo tiende a ir en aumento.

El nivel educativo tiende a ser más bien bajo. En el estudio de Arbinaga (2000) el 66,7\% de las ludópatas contaban sólo con el título de graduado escolar y el $33,3 \%$ no tenían estudios. Por tanto, se puede decir que, en general, cuanto menor es el nivel educativo, mayor es la probabilidad de desarrollar una adicción al juego.

Respecto a la situación laboral, hay un claro predominio de las amas de casa (más del $80 \%$ ) entre las ludópatas. El perfil más habitual es el del ama de casa que sale de casa para hacer la compra o resolver la intendencia del hogar y que se gasta el dinero en el juego (Becoña, 1997).

Cuando se corriparan los perfiles demográficos (edad, estado civil, estudios y nivel económico) de las ludópatas con los de los jugadores patológicos, no hay diferencias entre ellos, salvo en lo que se refiere a la ocupación laboral. Los ludópatas tienden a estar activos laboralmente, si bien en profesiones poco cualificadas.

\section{TIPOS DE JUEGO}

Hay ciertas diferencias en cuanto a los tipos de juegos de azar elegidos por los hombres y las mujeres. En general, los hombres tienden a jugar más a los juegos activos e inmediatos, como las máquinas tragaperras, y las mujeres a los pasivos y no tan inmediatos, como las loterías o los cupones (Becoña, 1997).

Sin embargo, por lo que a la ludopatfa propiamente se refiere, el bingo es el tipo de juego que está más presente en las mujeres ludópatas y, en menor medida, las máquinas tragaperras. Los bingos constituyen para muchas mujeres (el $60 \%$ de sus clientes son mujeres de más de 40 años) y Jubilados un centro de encuentro social. $\mathrm{Si}$ 
las mujeres han optado por autodenunciarse para no poder entrar en bingos y casinos, el riesgo de adicción a las máquinas tragaperras -presentes en muchos de los lugares recreativos- aumenta.

En EE UU los hombres tienden a engancharse a juegos que implican estrategia o competición con otras personas, como pueden ser el póker o el blackjack, mientras que las mujeres se decantan por juegos pasivos y que no impliquen relaciones interpersonales, como el bingo o las máquinas tragaperras (Hing y Breen, 2001).

\section{DESARROLLO DE LA LUDOPATÍA EN LA MUJER}

\section{Motlvaclones}

En general, los hombres tienden a jugar más por excitación y para ganar dinero. En estos casos la búsqueda de sensaciones, especialmente entre adolescentes, puede facilitar el contacto inicial con el juego y la impulsividad ser responsable de la persistencia en el juego, a pesar incluso de las pérdidas y de los problemas acumulados (cfr. Gupta y Deverensky, 1998).

Por el contrario, las mujeres tienden a recurrir al juego excesivo para hacer frente a problemas personales (soledad, aburrimiento, etcétera) y familiares (mala relación de pareja, marido enfermo o adicto al juego o al alcohol, etcétera), así como para afrontar un estado de ánimo distórico (depresión, ansiedad, ira, etcétera). El deseo de ver a otras personas, lo que no equivale necesariamente socializarse con ellas, en un lugar público puede ser una motivación adicional (Hing y Breen, 2001; Trevorrow y Moore, 1998).

Pero, por desgracia, estas diferencias motivacionales en uno y otro sexo no se han tenido en cuenta a la hora de diseñar tratamientos específicos (Raylu y Oei, 2002).

\section{Factores de rlesgo}

Los antecedentes familiares de juego o alcohol excesivo constituyen un factor de riesgo de ludopatía tanto para hombres como para 
mujeres (Arbinaga, 2001; Lesieur, 1993). El efecto de modelado de los padres es un factor de riesgo claro para que los adolescentes se inicien en el Juego, con las expectativas de la obtención de un dínero fácil.

En el caso de las mujeres adictas el riesgo aumenta cuando tienen antecedentes de un divorcio temprano de los padres, han sufrido sucesos traumáticos en la infancia, como abuso sexual o maltrato infantil, han experimentado pérdidas significativas (ruptura no deseada de la pareja, muerte de un hijo, etcétera) y cuentan con maridos con dependencia alcohólica o que se encuentran mucho tiempo fuera de casa. De hecho, muchas mujeres ludópatas comienzan a jugar como un modo de escape de problemas que las desbordan (soledad, maltrato, relaciones maritales problemáticas, etcétera) (Petry y Steinberg, 2005; Strachan y Custer, 1993).

En cuanto a las dimensiones de personalidad, las mujeres ludópatas tienden a mostrar puntuaciones altas en introversión. De hecho, la introversión se relaciona más con los juegos de azar pasivos y solitarios, como las loterías y los cupones, que son los más utilizados por las mujeres (cfr. Arbinaga, 2001; Femández-Montalvo y Echeburúa, 2001). Sin embargo, otras variables de personalidad significativas en los hombres ludópatas, como la impulsividad y la búsqueda de sensaciones, no parecen tan slgnificativas en las mujeres ludópatas. Más allá de dimensiones concretas, la soledad y la propensión al aburrimiento son variables que se relacionan estrechamente con la tendencia a la ludopatia. En estos casos el juego puede comenzar como un mero entretenimiento para ocupar el tiempo libre excesivo, como consecuencia del ínicio de los hijos en el colegio o de la salida de los hijos del hogar, y transformarse posteriormente en una adicción (Hing y Breen, 2001).

Respecto a los problemas psicopatológicos, la depresión primaria - la sintomatología ansioso-depresiva en la mujer son un factor de riesgo de implicación en la ludopatía, como lo son en los hombres ludópatas el abuso de alcohol y drogas y la implicación en conductas antisociales, lo que lleva frecuentemente a la existencia de antecedentes penales. Al ser más habitual la depresión en la mujer y al poderse utilizar el juego como una forma de escape de los problemas cotidianos a los que no se ve salida, el juego excesivo puede actuar 
Tabla 2.- Perfil dlferenclal de la ludopatía en el hombre y en la mujer

\begin{tabular}{|c|c|c|}
\hline & MUJER & HOMBRE \\
\hline $\begin{array}{l}\text { Preferenclas } \\
\text { de juego }\end{array}$ & $\begin{array}{l}\text { Bingo, máquinas } \\
\text { tragaperras, loterias } \\
\text { y cupones }\end{array}$ & $\begin{array}{l}\text { Máquinas } \\
\text { tragaperras, } \\
\text { casino y bingo }\end{array}$ \\
\hline Motivación & $\begin{array}{l}\text { Afrontamiento de } \\
\text { problemas } \\
\text { personales y del } \\
\text { estado de ánimo } \\
\text { disfórico }\end{array}$ & $\begin{array}{l}\text { Búsqueda de } \\
\text { sensaciones } \\
\text { Ganancia de } \\
\text { dinero }\end{array}$ \\
\hline $\begin{array}{l}\text { Dimenslones } \\
\text { de } \\
\text { personalldad }\end{array}$ & Introversión & $\begin{array}{l}\text { Impulsividad } \\
\text { Búsqueda de } \\
\text { sensaciones }\end{array}$ \\
\hline $\begin{array}{l}\text { Factores de } \\
\text { rlesgo }\end{array}$ & $\begin{array}{l}\text { Depresión primaria } \\
\text { Sintomatología } \\
\text { ansioso-depresiva } \\
\text { Multiimpulsividad } \\
\text { Víctima de abuso en } \\
\text { la infancla }\end{array}$ & $\begin{array}{l}\text { Abuso de alcohol } \\
\text { y drogas } \\
\text { Conductas } \\
\text { antisociales }\end{array}$ \\
\hline $\begin{array}{l}\text { Evolución del } \\
\text { trastorno }\end{array}$ & $\begin{array}{l}\text { Inicio tardío } \\
\text { Progrestón más } \\
\text { rápida }\end{array}$ & $\begin{array}{l}\text { Iniclo anterior } \\
\text { Progresión más } \\
\text { lenta }\end{array}$ \\
\hline $\begin{array}{l}\text { Comorbilldad } \\
\text { asociada }\end{array}$ & $\begin{array}{l}\text { Depresión } \\
\text { Problemas } \\
\text { psicosomáticos } \\
\text { Episodios bulímicos }\end{array}$ & $\begin{array}{l}\text { Depresión } \\
\text { secundaria } \\
\text { Problemas con la } \\
\text { justicia } \\
\text { Conductas } \\
\text { temerarias } \\
\end{array}$ \\
\hline $\begin{array}{l}\text { Búsqueda de } \\
\text { ayuda } \\
\text { terapéutica }\end{array}$ & $\begin{array}{l}\text { En solitarlo } \\
\text { Actltud de } \\
\text { vergüenza }\end{array}$ & $\begin{array}{l}\text { En compañía de } \\
\text { su pareja } \\
\text { Actitud altiva o } \\
\text { arrogante }\end{array}$ \\
\hline
\end{tabular}


en la mujer como una fuente de activación para superar el estado de ánimo deprimido. En el hombre ludópata, en cambio, es más habitual la depresión secundaria a la ludopatía, ligada habitualmente a las consecuencias negativas del juego excesivo.

Otro factor predisponente para la ludopatía en la mujer es la multiimpulsividad, que se pone de relieve en conductas corno la presencia de atracones o de episodios bulímicos, el abuso de alcohol, el fumar compulsivo, el consumo exagerado de fármacos o la adicción al sexo (Becoña, 1997).

Los factores precipitantes más habituales en las mujeres ludópatas son el consumismo excesivo, que puede incitar a la búsqueda de un dinero fácil en el juego (especialmente cuando se trata de personas supersticiosas o con una distorsión cognitiva de la realidad), la estancia habitual en los bares como consecuencia de la profesión o de la afición y la frecuentación de amigos jugadores o el establecimiento de una relación de pareja con un jugador excesivo (tabla 2).

\section{Evoluclón del trastorno}

El curso de la ludopatía en la mujer es, en muchos aspectos, muy similar al del varón ludópata. Sin embargo, hay algunos aspectos diferenciales en los que merece la pena reparar.

En cuanto a la edad de inicio, y como ocurre también en otras conductas adictivas (abuso de alcohol, tabaquismo, etcétera), las mujeres comienzan a jugar más tarde que los hombres. En lugar de empezar a hacerlo en la adolescencia, hay un grupo de mujeres que se inician en el juego entre los 30 y 40 años de edad, cuando se complican las circunstancias vitales (soledad, mala relación de pareja, problemas con los hijos, etcétera). Por ello, la ludopatía aparece en la mujer en las edades medias o tardias de la vida (Becoña, 1997), controlada fundamentalmente por reforzadores negativos (evitación del malestar emocional y escape de las frustraciones cotidianas).

Es en el curso del trastorno en donde hay unas diferencias más significativas entre hombres y mujeres. Las mujeres se inician más 
tardíamente en el juego, pero, sin embargo, la progresión hacia el trastorno es dos veces más rápida que en el caso del hombre. En concreto, la evolución del trastomo antes de la búsqueda de ayuda terapéutica es de 11 años en el caso de los hombres y de 4 en el de las mujeres (Ibáñez, Blanco, Moreryra y Saiz, 2003; Tavares, Zilberman, Beites y Gentil, 2001).

El desarrollo del trastomo es más rápido cuando las mujeres afectadas carecen de habilidades de autocontrol, de comunicación y de solución de problemas, cuando su apoyo social es escaso y la utilización del tiempo libre poco satisfactoria y cuando cuentan con pocos recursos psicológicos para hacer frente a las situaciones de estrés.

El curso de la ludopatía está asociado a la aparición de alteraciones mentales (depresión) o de problemas psicosomáticos (fatiga crónica, dolores de cabeza, alteraciones gastrointestinales, etcétera) mucho más frecuentemente que en el caso del hombre. Sin embargo, los problemas con la justicia por robos, falsificaciones, préstamos no devueltos, etcétera, son menos habituales que en el caso del hombre. Por asi decirlo, la evolución de la ludopatía en la mujer cursa de una forma más interna y menos visible.

\section{Factores de contenclón}

El rechazo social a la mujer adicta (sea alcohólica o toxicómana, sea ludópata) está mucho más arraigado que el experimentado por el hombre en esas mismas circunstancias. Es más, el apoyo familiar de la pareja o de los hijos hacia la mujer adicta es mucho menor que el recibido por el hombre adicto, al que se considera más como enfermo que como vicioso, al revés de lo que ocurre en la mujer jugadora.

La interiorización de este rechazo hacia las mujeres adictas o hacia la presencia regular de las mujeres en los bares (lugar habitual de las máquinas tragaperras) es una barrera de contención que frena la progresión en el juego a muchas mujeres (Becoña, 1997).

Asimismo las mujeres suelen tener un mayor control en la obtención y en el manejo del dinero. En general, al menos tradicional- 
mente, al ser amas de casa o tener profesiones menos cualificadas, cuentan con menos dinero propio y, por ello, se entrampan menos, lo que facilita una contención de la espiral de deterioro.

Por último, la relevancia de la mujer como madre de los hijos y la responsabilidad familiar y social inherente a ello suponen un freno en la progresión de la ludopatía.

\section{CONSECUENCIAS DE LA LUDOPATÍA EN LA MUJER}

\section{A nivel físico}

Las alteraciones psicosomáticas, que la llevan a consultar a los médicos de atención primaria, y los problemas de salud mental, como la depresión o síntomas inespecíficos de ansiedad, constituyen la parte más visible de la ludopatía en la mujer y pueden contribuir a ocultar el problema de raíz (la ludopatía).

\section{A nivel psicológico}

El sufrimiento causado por las pérdidas económicas y la inseguridad permanente de ser descubierta en su adicción generan en la mujer ludópata un estado de irritabilidad, de nerviosismo y de inestabilidad emocional que acaba por aislarla familiar y socialmente. Además las mentiras y los autoengaños deterioran la autoestima de la mujer ludópata.

Todo ello acentúa una sensación de inutilidad y facilita la aparición de un estado de ánimo deprimido, que, en ocasiones, es preexistente, pero que, en otras, es resultado de la degradación personal y del rechazo familiar y social que experimenta la mujer como consecuencia de la adicción.

\section{A nivel familiar, social y profesional}

La reacción del marido o de la pareja ante la mujer ludópata es mucho más intransigente que cuando sucede al revés (Custer y Milt, 
1985). En este contexto resulta habitual la violencia de pareja. La consideración de la mujer ludópata como viciosa, algo similar a como si fuese una alcohólica o una prostituta, denota un enfoque moral del problema, no en términos de salud/enfermedad. El descuido de los hijos y la denivación del dinero familiar hacia el juego acentúan este enfoque (cfr. Becoña, 1997).

La repercusión negativa de la ludopatía en la mujer es tanto mayor cuanto más pequeños sean los hijos. En concreto, existe un mayor riesgo de abuso paterno contra los hijos y son más frecuentes en éstos trastomos de conducta, alteraciones psicosomáticas, ausencias del hogar, fracaso escolar, consumo de drogas y de juego y conductas asociales (Becoña, 1997; Jacobs, 1989). En estos casos, a un modelo materno inadecuado se une frecuentemente la inestabilidad familiar.

Cuando la mujer ludópata es ama de casa, se produce a menudo una desatención a las tareas del hogar y una inversión del dinero familiar en el juego. Si se trata de una mujer profesional, la disminución del rendimiento laboral, con inasistencias o retrasos e incumplimiento de las tareas profesionales, es la conducta habitual, lo que pone en peligro su estabilidad laboral.

Por último, la situación se agrava con el aislamiento social de la mujer ludópata, que es resultado del intento de la mujer de ocultar su realidad ante otras personas y del rechazo social de las personas de su entorno. Todo ello contribuye a aumentar sus emociones negativas.

\section{COMORBILIDAD ASOCIADA}

La depresión, con el riesgo consiguiente de suicidio, es el trastorno comórbido más frecuente en la mujer jugadora, sobre todo cuando la ludopatía entraña ya una fase de deterioro (lbáñez et al., 2003). Por lo que a la diferencia de sexos se refiere, la depresión puede ser un factor predisponte al juego en la mujer, que luego se acentúa con el impacto desfavorable del juego, mientras que en el hombre la depresión puede ser secundaria a la ludopatla, ligada a sus consecuencias negativas de toda indole. 
La depresión es más habitual cuando hay violencia de pareja o cuando la mujer se siente desbordada por las responsabilidades familiares (educación de los hijos, situación económica precaria, rechazo de la pareja, etcétera).

Asimismo el abuso de alcohol es una conducta de riesgo en la ludópata, sobre todo cuando ya se ha establecido una dependencia acentuada al juego (Arbinaga, 2001).

Los ludópatas de uno y otro sexo manifiestan conductas impulsivas asociadas al juego. Desde una perspectiva diferencial, los hombres tienden a implicarse más en conductas temerarias y de abuso de aicohol; las mujeres, en trastornos psicosomáticos, en conductas suicidas ligadas a la depresión y en episodios bulímicos (lbáñez et al., 2003; Martins, Tavares, Lobo, Galetti y Gentil, 2004).

\section{TRATAMIENTO}

El estigma social negativo, especialmente acentuado en el caso de la mujer, contribuye a impedir el reconocimiento del problema y a retrasar la búsqueda de ayuda terapéutica. Es, con frecuencia, el deterioro de la relación familiar la variable crítica que determina la consulta por la ludopatía.

La búsqueda de ayuda terapéutica por parte de la mujer ludópata se hace generalmente en solitario. A diferencia del hombre ludópata, que suele venir acompañado por alguno de sus familiares cercanos, la mujer acude sola y no cuenta con una colaboración activa por parte de su pareja en el tratamiento.

Asimismo, y a diferencia del hombre ludópata, que adopta una actitud más egocéntrica, altiva y negadora del problema, la mujer ludópata, una vez que ha dado el paso inicial de buscar ayuda, es más consciente de lo ocurrido y muestra un profundo sentimiento de vergũenza (Becoña, 1997).

\section{CONCLUSIONES}

La dependencia al juego se da tanto en hombres como en mujeres. Si bien es más frecuente (en una proporción de $7 / 3$ ) en los hombres 
que en las mujeres, éstas son mucho más reacias a reconocer el problema y a buscar ayuda terapéutica. De hecho, no más del $10 \%$ - 15\% de ludópatas en tratamiento son mujeres.

Se sabe que la mujer ludópata presenta un perfil diferencial en cuanto a los tipos de juegos implicados, su historia de juego y las manifestaciones psicosociales de su dependencia. Sin embargo, la limitación de los hallazgos obtenidos hasta la fecha deriva, en buena medida, de los escasos trabajos publicados en que se ha estudiado específicamente este tema.

Los principales retos de futuro en este ámbito son diseñar estrategias motivacionales para atraer a las mujeres al tratamiento, contar con instrumentos de detección temprana (de aplicación, por ejemplo, en el ámbito de los Centros de Atención Primaria, dada la presencia de muchos sintomas psicosomáticos en las mujeres ludópatas) y desarrollar programas de tratamiento encaminados específicamente a los déficit de conducta experimentados por las jugadoras patológicas.

Por último, una línea sugerente de investigación es poner a punto programas de intervención para las mujeres acompañantes de los ludópatas (parejas, hijas o madres), de modo que su labor para atraer al tratamiento a los pacientes directos o su actuación como coterapeutas sean lo más eficaces posible.

\section{BIBLIOGRAFIA}

Arbinaga, F. (2000). Características sociodemográficas, consumo de drogas, depresión y juego patológico en un grupo de mujeres de Punta Umbría (Huelva): Un estudio descriptivo. Anales de Psicolog/a, 16, 123-132.

Arbinaga, F. (2001). La mujer con problemas en los juegos de apuesta: una aproximación desde los estudios epldemiológicos realizados en nuestro país y el análisis de nuevos datos sobre un grupo de mujeres en Punta Umbria (Huelva). Psicologemas, 15, 133-152.

Báez, C., Echeburúa, E. y Fernández-Montalvo, J. (1994). Características demográficas, de personalidad y psicopatológicas de los jugadores 
patológicos de máquinas tragaperras en tratamiento: un estudio descriptivo. Clinica y Salud, 5, 289-305.

Báez, C., Echeburúa, E. y Fernández-Montalvo, J. (1995). Variables predictoras de abandonos y recardas en el tratamiento del juego patológico. Análisis y Modificación de Conducta, 21, 5-22.

Becoña, E. (1997). Características de la mujer jugadora patológica. Revista de Psicopatología y Psicología Clínica, 2, 21-34.

Becoña, E. (1999). Epidemiología del juego patológico en España. Anuario de Psicologla, 30, 7-19.

Custer, R.L. y Mllt, H. (1985). When lucks runs out. New York. Facts on File Publications.

Echeburúa, E. (1992). Psicopatología, variables de personalidad y vulnerabilidad psicológica al juego patológico. Psicothema, 4, 7-20.

Echeburúa, E., Fernández-Montalvo, J. y Báez, C. (1999a). Avances en el tratamiento del juego patológico. Adicciones, 11, 349-361.

Echeburúa, E. y Femández-Montaivo, J. (2003). Nuevas perspectivas en el tratamiento del juego patológico. Revista Thomson de Psicologla, 1, 139-155.

Fernández-Montalvo, J. y Echeburúa, E. (2001). Trastornos de personalidad y juego patológico: una revisión crítica. Psicologla Conductual, 9, 527-539.

Getty, H., Watson, J. y Frlsch, G. (2000). A Comparison of Depression and Styles of Coping in Male and Female GA Members and Controls. Joumal of Gambling Studies, 16, 377-391.

Grant, J. y Won KIm, S. (2002). Gender Differences in Pathological Gamblers Seeking Medication Treatment. Comprehensive Psychlatry, 43, 56-62.

Gupta, R. y Deverensky, J. (1998). Adolescent Gambling Behaviour: A prevalence Study and Examination of the Correlatos Associated with Problem Gambling. Journal of Gambling Studies, 14, 319-343.

Hing, N. y Breen, H. (2001). Profiling Lady Luck: An Empirical Study of Gambling and Problem Gambling Amongst Female Club Members. Joumal of Gambling Studies, 17, 47-69.

Ibáñez, A., Blanco, C., Moreryra, P. y Salz-Rulz, J. (2003). Gender Differences in Pathological Gambling. Joumal of Clinical Psychiatry, 64, 295-301.

Irurlte, I.M. (1996). Estudio sobre la prevalencla de los jugadores de azar en Andalucia. Sevilla. Comisionado para la Droga, Consejerla de Asuntos Sociales, Junta de Andalucía.

Jacobs, D.F. (1989). A general theory of addictions: Rationale for an evidence supporting a new approach for understanding and treating addictive behaviors. In H.J. Shaffer, S.A. Stein, B. Gambino y T.N. Cummings 
(Eds.). Compulsive gambling. Theory, research and practice. Lexington, MA. Lexington Books.

Ladd, G. y Petry, N. (2002). Gender Differences Among Pathological Gamblers Seeking Treatment. Experimental and Clinical Psychopharmacology, 10, 302-309.

Lesleur, H.R. (1993). Prevalencia, caracteristicas y tratamiento de los jugadores en los Estados Unidos. Psicología Conductual, 1, 389-407.

Lesieur, H.R. y Helneman, M. (1988). Pathological gambling among youthful multiple substance abusers in a therapeutic community. British Joumal of Addictions, 83, 765-771.

MartIns, S., Tavares, H., Lobo, D., Galettl, A. y Gentil, V. (2004). Pathological gambling, gender, risk-taking behaviors. Addictive Behaviors, 29, 1231-1235.

McCormlck, R.A., Russo, A., Ramírez, L y Taber, J. (1984). Affective disorders among pathological gamblers seeking treatment. American Joumal of Psychiatry, 141, 215-218.

Petry, N. y Stelnberg, K. (2005). Childhood Maltreatment in Male and Female Treatment-Seeking Pathological Gamblers. Psychology of Addictive Behaviors, 19, 226-229.

Raylu, N. y Oel, T. (2002). Pathological gambling. A comprehensive review. Clinical Psychology Review, 22, 1009-1061.

Rodríguez-Martos, A. (1989). Estudio piloto estimativo de la prevalencia de juego patológico entre los pacientes alcohólicos que acuden al Programa DROSS. Revista Española de Drogodependencias, 14, 265-275.

Strachan, M.L. y Custer, R.L. (1993). Female compulsive gamblers in Las Vegas. In W.R. Eadington y J.A. Cornelius (Eds.). Gambling Behavior \& Problem Gambling. Reno, NV. Institute for the Study of Gambling and Commercial Gaming.

Tavares, H., Zllberman, M., Beltes, F. y Gentil, V. (2001). Gender differences in gambling progression. Joumal of Gambling Studies, 17, 151-159.

Tavares, H., Martins, S., Lobo, D., Sllvelra, C., Gentil, V. y Hodgins, D. (2003). Factors at Play in Faster Progression for Female Pathological Gamblers: An Exploratory Analysis. Joumal of Clinical Psychiatry, 64, 433-438.

Tejelro, R. (1996). Estudio sobre el juego de azar en Algeciras. Prevalencia y características sociodemográficas. Algeciras. JARCA.

Trevorrow, K. y Moore, S. (1998). The Association Between Loneliness, Social Isolation and Women's Electronic Gaming Machine Gambling. Joumal of Gambling Studies, 14, 263-284. 